\title{
Dynamics of an Infectious Disease Including Ectoparasites, Rodents and Humans
}

\author{
A. Dénes and G. Röst
}

\section{Introduction}

Ectoparasites are parasites that live on or in the skin but not within the body. These parasites, e.g. lice, fleas, mites have long been known as vectors of several infectious diseases including epidemic typhus and plague. It is also commonly known that in several cases, ectoparasites are transmitted to humans from animals, most often by rodents. A well-known example for this is plague, caused by the bacterium Yersinia pestis: the fleas transmitting this disease were transmitted to humans by rats [5]. Other notable examples are Omsk haemorrhagic fever, caused by a Flavivirus transmitted by ticks on water voles and muskrats [4]; rickettsialpox, caused by the bacteria Rickettsia akari transmitted by mites on mice [6]; murine typhus, caused by the bacteria Rickettsia typhi, transmitted by fleas, usually on rats [9]; scrub typhus caused by the parasite Orientia tsutsugamushi, transmitted by trombiculid mites, carried by mice. The latter disease is estimated to cause more than a million cases annually in Asia with more than a billion people being at risk, which makes scrub typhus the most medically important rickettsial disease [10] (Figs. 1 and 2).

In this work, we consider an infectious disease caused by a pathogen spread by ectoparasites which are harboured by rodents. We assume that ectoparasites spread by the rodents might be infectious or non-infectious. A given rodent or human can be infested only by one type (either infectious or non-infectious) of the ectoparasite. A human can be infested (and hence possibly infected) through adequate contact with an infested (infected) rodent or another human. We assume that the ectoparasites are not transmitted back from humans to the rodents. Due to infestation and/or treatment, infested and infected humans may become susceptible again.

\footnotetext{
A. Dénes $(\bowtie) \cdot$ G. Röst

Bolyai Institute, University of Szeged, Szeged, Hungary

e-mail: denesa@math.u-szeged.hu
} 
Fig. 1 The pathogen can jump from rodents to humans via ectoparasites. Figure: courtesy of Júlia Röst
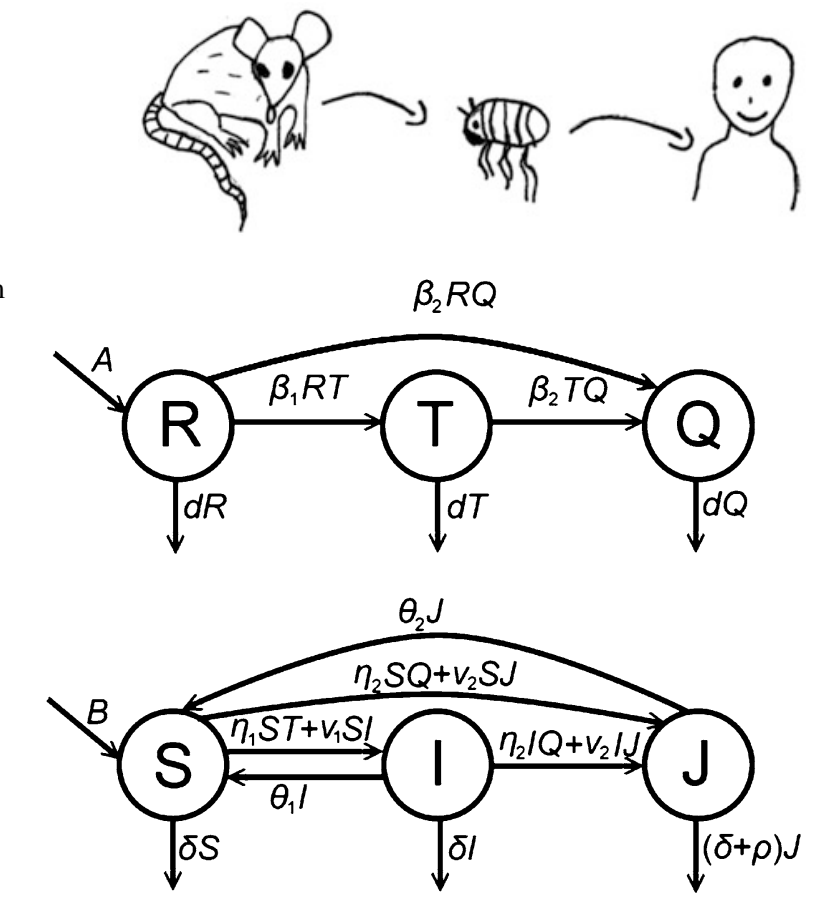

Fig. 2 Transmission diagram representing transitions between the rodent and the human compartments

The structure of the paper is as follows. In Sect. 2, we establish a compartmental model describing the spread of the infestation and the disease. In Sect. 3, we study the subsystem formed by the equations for the rodent compartments, while, using the results of Sect. 3, we study the human subsystem in Sect. 4.

\section{The Model}

We denote by $R(t)$ the compartment of susceptible rodents, $T(t)$ stands for the rodents infested by non-infectious parasites, while $Q(t)$ denotes the number of rodents infested by infectious parasites. Similarly, we have three compartments for the humans: $S(t)$ denotes susceptibles, $I(t)$ those infested by non-infectious parasites, and $J(t)$ those infested by infectious parasites. $A$ and $d$ stand for the birth, resp. death rates of rodents. The notation $\beta_{1}$ stands for the transmission rate between the compartments $R$ and $T$, while $\beta_{2}$ is the transmission rate between $R$ and $Q$, resp. $T$ and $Q . B$ and $\delta$ stand for natural birth and death rates for humans, and $\rho$ denotes disease-induced death rate for the infected human compartment $J$. The parameter $v_{1}$ denotes transmission rate between the compartments $S$ and $I$, while $v_{2}$ denotes transmission rate from $J$ to $S$ and $J$ to $I$. The parameter $\eta_{1}$ denotes the transmission rate from rodents infested by non-infectious parasites to susceptible humans, while 
$\eta_{2}$ is the transmission rate from rodents infested by infectious parasites to humans. We denote by $\theta_{1}$, resp. $\theta_{2}$ the disinfestation, resp. recovery rate from compartments $I$, resp. $J$.

Using the above notations, our equations take the following form:

$$
\begin{aligned}
R^{\prime}(t)= & A-\beta_{1} R(t) T(t)-\beta_{2} R(t) Q(t)-d R(t), \\
T^{\prime}(t)= & \beta_{1} R(t) T(t)-\beta_{2} T(t) Q(t)-d T(t), \\
Q^{\prime}(t)= & \beta_{2} R(t) Q(t)+\beta_{2} T(t) Q(t)-d Q(t), \\
S^{\prime}(t)= & B-\eta_{1} S(t) T(t)-\eta_{2} S(t) Q(t)-v_{1} S(t) I(t)-v_{2} S(t) J(t) \\
& -\delta S(t)+\theta_{1} I(t)+\theta_{2} J(t), \\
I^{\prime}(t)= & \eta_{1} S(t) T(t)+v_{1} S(t) I(t) \\
& -\eta_{2} I(t) Q(t)-v_{2} I(t) J(t)-\delta I(t)-\theta_{1} I(t), \\
J^{\prime}(t)= & \eta_{2} S(t) Q(t)+\eta_{2} I(t) Q(t)+v_{2} S(t) J(t)+v_{2} I(t) J(t) \\
& -\delta J(t)-\rho J(t)-\theta_{2} J(t),
\end{aligned}
$$

with positive initial conditions $R(0), T(0), Q(0), S(0), I(0), J(0) \geq 0$. The phase space

$$
\mathbb{R}_{+}^{6}=\left\{(R, T, Q, S, I, J) \in \mathbb{R}^{6}: R, T, Q, S, I, J \geq 0\right\}
$$

is clearly invariant to system (1).

\section{The Rodent Subsystem}

\subsection{Equilibria, Local Stability}

The first three equations of (1) can be decoupled from the remaining ones. The subsystem for the spread among rodents, given by

$$
\begin{aligned}
& R^{\prime}(t)=A-\beta_{1} R(t) T(t)-\beta_{2} R(t) Q(t)-d R(t), \\
& T^{\prime}(t)=\beta_{1} R(t) T(t)-\beta_{2} T(t) Q(t)-d T(t), \\
& Q^{\prime}(t)=\beta_{2} R(t) Q(t)+\beta_{2} T(t) Q(t)-d Q(t)
\end{aligned}
$$

has a similar structure as the model given by Dénes and Röst [1, 2], though, in the present case, birth and death rates are not equal in contrast to the cited papers. To calculate the equilibria of the full system, we start by calculating those of the rodent subsystem (2), which are easily obtained by solving the algebraic system of equations 


$$
\begin{aligned}
& 0=A-\beta_{1} R T-\beta_{2} R Q-d R, \\
& 0=\beta_{1} R T-\beta_{2} T Q-d T, \\
& 0=\beta_{2} R Q+\beta_{2} T Q-d Q,
\end{aligned}
$$

resulting in the four possible equilibria

$$
\begin{array}{ll}
E_{R}=\left(\frac{A}{d}, 0,0\right), & E_{T}=\left(\frac{d}{\beta_{1}}, \frac{A}{d}-\frac{d}{\beta_{1}}, 0\right) \\
E_{Q}=\left(\frac{d}{\beta_{2}}, 0, \frac{A}{d}-\frac{d}{\beta_{2}}\right), & E_{T Q}=\left(\frac{A \beta_{2}}{d \beta_{1}}, \frac{d}{\beta_{2}}-\frac{A \beta_{2}}{d \beta_{1}}, \frac{A}{d}-\frac{d}{\beta_{2}}\right) .
\end{array}
$$

By introducing a single infested/infected individual into one of the equilibria $E_{R}$, $E_{T}$ and $E_{Q}$, we obtain three different reproduction numbers. If we introduce a rodent infested by the non-infectious parasites into the disease- and infestation-free equilibrium, we obtain the reproduction number $r_{1}=\frac{A \beta_{1}}{d^{2}}$.

Introducing a rodent infested by the infectious parasites into the equilibrium $E_{R}$, we obtain the reproduction number $r_{2}=\frac{A \beta_{2}}{d^{2}}$.

If we introduce a rodent infested by the infectious parasites into the equilibrium $E_{T}$, we obtain again the same reproduction number $r_{2}$. Finally, let us introduce a rodent infested by the non-infectious parasites into the equilibrium $E_{Q}$. In this case, the expected sojourn time of an individual infected with the first strain in the $T$ compartment is $\left(\beta_{2} Q^{*}+d\right)^{-1}$, and the number of new infections generated by this individual is $\beta_{1} R^{*}$, where $R^{*}$ and $Q^{*}$ stand for the first, resp. third coordinates of the equilibrium $E_{Q}$. This way we obtain the reproduction number $r_{3}=\frac{\beta_{1} d^{2}}{\beta_{2}^{2} A}$.

It is obvious that the equilibrium $E_{R}$ always exists, $E_{T}$ exists if and only if $r_{1}>$ $1, E_{Q}$ exists if and only if $r_{2}>1$, while $E_{T Q}$ exists if and only if $r_{2}>1$ and $r_{3}>1$.

The following proposition on the local stability of the four equilibria can easily be checked, see [3].

Proposition 3.1 The disease-free equilibrium $E_{R}$ is locally asymptotically stable if $r_{1}<1$ and $r_{2}<1$ and unstable if $r_{1}>1$ or $r_{2}>1$. The equilibrium $E_{T}$ is locally asymptotically stable if $r_{1}>1$ and $r_{2}<1$. The equilibrium $E_{Q}$ is locally asymptotically stable if $r_{2}>1$ and $r_{3}<1$. The equilibrium $E_{T Q}$ is locally asymptotically stable if $r_{2}>1$ and $r_{3}>1$.

\subsection{Persistence}

Before we can state our results on the persistence of the three compartments, we will need some notions and theorems from [8].

Definition 3.1 Let $X$ be a nonempty set and $\rho: X \rightarrow \mathbb{R}_{+}$. A semiflow $\Phi: \mathbb{R}_{+} \times$ $X \rightarrow X$ is called uniformly weakly $\rho$-persistent, if there exists some $\varepsilon>0$ such that $\lim \sup _{t \rightarrow \infty} \rho(\Phi(t, x))>\varepsilon$ for all $x \in X, \rho(x)>0$. $\Phi$ is called uniformly 
(strongly) $\rho$-persistent if there exists some $\varepsilon>0$ such that $\liminf _{t \rightarrow \infty} \rho(\Phi(t, x))>$ $\varepsilon$ for all $x \in X, \rho(x)>0$. A set $M \subseteq X$ is called weakly $\rho$-repelling if there is no $x \in X$ such that $\rho(x)>0$ and $\Phi(t, x) \rightarrow M$ as $t \rightarrow \infty$.

System (2) generates a continuous flow on the phase space

$$
X:=\left\{(R, T, Q) \in \mathbb{R}_{+}^{3}\right\} .
$$

Theorem 3.1 $R(t)$ is always uniformly persistent. $T(t)$ is uniformly persistent if $r_{1}>1$ and $r_{2}<1$ as well as if $r_{2}>1$ and $r_{3}>1$. $Q(t)$ is uniformly persistent if $r_{2}>1$.

Proof To show uniform persistence of the susceptible compartment, we will use the method of fluctuation (see, e.g., [7, Lemma A.1]). We denote by $R_{\infty}$ the limit inferior of $R(t)$, while $T^{\infty}$ and $Q^{\infty}$ denote the limit superior of $T(t)$, resp. $Q(t)$ as $t \rightarrow \infty$. Using the fluctuation lemma we know that there exists a time sequence $t_{k} \rightarrow \infty$ such that $R\left(t_{k}\right) \rightarrow R_{\infty}$ and $R^{\prime}\left(t_{k}\right) \rightarrow 0$ as $k \rightarrow \infty$. If we apply this to the equation for $R(t)$, we obtain

$$
R^{\prime}\left(t_{k}\right)+\beta_{1} R\left(t_{k}\right) T\left(t_{k}\right)+\beta_{2} R\left(t_{k}\right) Q\left(t_{k}\right)=A .
$$

It is easy to see that for the total rodent population we have $R(t)+T(t)+Q(t) \rightarrow \frac{A}{d}$, thus, $0 \leq T^{\infty} \leq \frac{A}{d}$ and $0 \leq Q^{\infty} \leq \frac{A}{d}$. Using this and letting $k \rightarrow \infty$ we get $R_{\infty} \geq \frac{d}{\beta_{1}+\beta_{2}}$.

To show persistence of the infested compartments, we need some theory from [8]. We use the notation $x=(R, T, Q) \in X$ for the state of the system and the usual notation $\omega(x)$ for the $\omega$-limit set of a point $x$ defined as

$$
\omega(x):=\left\{y \in X: \exists\left\{t_{n}\right\}_{n \geq 1} \text { s. t. } t_{n} \rightarrow \infty \text { and } \Phi\left(t_{n}, x\right) \rightarrow y \text { as } n \rightarrow \infty\right\} .
$$

We first show the persistence of $T(t)$. Let $\rho(x)=T$. Let us consider the invariant extinction space of $T$, defined as $X_{T}:=\{x \in X: \rho(x)=0\}$. We follow [8, Chapter 8] and examine the set $\Omega_{x \in X_{T}}:=\cup_{x \in X_{T}} \omega(x)$. Applying the BendixsonDulac criterion with Dulac function $1 / Q$ and the Poincaré-Bendixson theorem, we obtain that all solutions in the extinction space $X_{T}$ tend to an equilibrium.

Let us first consider the case $r_{1}>1$ and $r_{2} \leq 1$. Clearly, in this case $\Omega=\left\{E_{R}\right\}$. As a first step, we prove weak $\rho$-persistence. In order to apply [8, Theorem 8.17], we let $M_{1}=\left\{E_{R}\right\}$. Then $\Omega$ is a subset of $M_{1}$, which is isolated, compact, invariant and acylic. We have to show that $M_{1}$ is weakly $\rho$-repelling, from which we obtain persistence.

Let us suppose that this does not hold, i.e. there exists a solution such that $\lim _{t \rightarrow \infty}(R(t), T(t), Q(t))=\left(\frac{A}{d}, 0,0\right)$ and $T(t)>0$. Then for any $\varepsilon>0$, for sufficiently large $t$, we have $R(t)>\frac{A}{d}-\varepsilon$ and $Q(t)<\varepsilon$. For such $t$, we can give the following estimation for $T^{\prime}(t)$ : 


$$
T^{\prime}(t)=T(t)\left(\beta_{1} R(t)-\beta_{2} Q(t)-d\right)>T(t)\left(\beta_{1} \frac{A}{d}-\beta_{1} \varepsilon-\beta_{2} \varepsilon-d\right),
$$

which is positive if $\varepsilon$ is sufficiently small as $\frac{A \beta_{1}}{d}>d$ follows from $r_{1}>1$. This contradicts $T(t) \rightarrow 0$.

In the second case, when $r_{2}>1$ and $r_{3}>1$, also $E_{Q}$ exists, so we have $\Omega=$ $\left\{E_{R}, E_{Q}\right\}$. Now we let $M_{1}=\left\{E_{R}\right\}$ and $M_{2}=\left\{E_{Q}\right\}$. Clearly, $\Omega \subset M_{1} \cup M_{2}$ and $\left\{M_{1}, M_{2}\right\}$ is acyclic and $M_{1}$ and $M_{2}$ are invariant, compact and isolated. We have to show that $M_{1}$ and $M_{2}$ are weakly $\rho$-repelling.

Suppose first that $M_{1}$ is not weakly $\rho$-repelling. Then there exists a solution such that $\lim _{t \rightarrow \infty}(R(t), T(t), Q(t))=\left(\frac{A}{d}, 0,0\right)$ and $T(t)>0$. Again, for any $\varepsilon>0$, for sufficiently large $t$, we have $R(t)>\frac{A}{d}$ and $Q(t)<\varepsilon$ and for such $t$, we can give the following estimation for $T^{\prime}(t)$ :

$$
T^{\prime}(t)=T(t)\left(\beta_{1} R(t)-\beta_{2} Q(t)-d\right)>T(t)\left(\beta_{2} \frac{A}{d}-\beta_{1} \varepsilon-\beta_{2} \varepsilon-d\right),
$$

where we used that $\beta_{1}>\beta_{2}$, which follows from $r_{2} r_{3}>1$. This expression is positive for $\varepsilon$ small enough, which contradicts $T(t) \rightarrow 0$.

Now let us suppose that $M_{2}$ is not weakly $\rho$-repelling. Then there exists a solution such that $\lim _{t \rightarrow \infty}(R(t), T(t), Q(t))=\left(\frac{d}{\beta_{2}}, 0, \frac{A}{d}-\frac{d}{\beta_{2}}\right)$. Then, for any $\varepsilon>0$, if $t$ is large enough, then $R(t)>\frac{d}{\beta_{2}}-\varepsilon$ and $Q(t)<\frac{A}{d}-\frac{d}{\beta_{2}}+\varepsilon$ and for such $t$ we can give the following estimation for $T^{\prime}(t)$ :

$$
\begin{aligned}
T^{\prime}(t) & =T(t)\left(\beta_{1} R(t)-\beta_{2} Q(t)-d\right) \\
& >T(t)\left(\frac{d \beta_{1}}{\beta_{2}}-\beta_{1} \varepsilon-\beta_{2}\left[\frac{A}{d}-\frac{d}{\beta_{2}}+\varepsilon\right]-d\right) \\
& =T(t)\left(\frac{d \beta_{1}}{\beta_{2}}-\frac{A \beta_{2}}{d}-\left(\beta_{1}+\beta_{2}\right) \varepsilon\right),
\end{aligned}
$$

which is positive for $\varepsilon$ small enough as $r_{3}>1$. This contradicts $T(t) \rightarrow 0$.

Let us now turn to the persistence of $Q(t)$ in the case $r_{2}>1$. We set $\rho(x)=Q$. We have the equilibrium $E_{R}$ if $r_{1} \leq 1$ and the two equilibria $E_{R}$ and $E_{T}$ if $r_{1}>1$. Similarly to the case of $T(t)$, we define the extinction space of $Q$ as $X_{Q}:=\{x \in$ $X: \rho(x)=0\}=\left\{(R, T, 0) \in \mathbb{R}_{+}^{3}\right\}$. In this case we have $\Omega=\cup_{x \in X_{Q}} \omega(x)=\left\{E_{R}\right\}$ if $r_{1} \leq 1$ and $\Omega=\cup_{x \in X_{Q}} \omega(x)=\left\{E_{R}, E_{T}\right\}$ if $r_{1}>1$. We define $M_{1}=\left\{E_{R}\right\}$ and $M_{2}=\left\{E_{T}\right\}$. Just like in the proof of the persistence of $T(t), \Omega$ is invariant, and $M_{1}$ and $M_{2}$ are isolated and acyclic.

To show that $M_{1}$ is weakly $\rho$-repelling, we can proceed in an analogous way as in the case of $T(t)$.

In the case $r_{1}>1$, we have to show that $M_{2}$ is weakly $\rho$-repelling. Suppose this does not hold. Then there exists a solution such that $\lim _{t \rightarrow \infty}(R(t), T(t), Q(t))=$ $\left(\frac{d}{\beta_{1}}, \frac{A}{d}-\frac{d}{\beta_{1}}, 0\right)$ and $Q(t)>0$. Then, for any $\varepsilon>0$, if $t$ is sufficiently large, then $R(t)>\frac{d}{\beta_{1}}-\varepsilon$ and $T(t)>\frac{A}{d}-\frac{d}{\beta_{1}}-\varepsilon$ and for such $t$ we can give the following estimation for $Q(t)$ : 


$$
\begin{aligned}
Q^{\prime}(t) & =Q(t)\left(\beta_{2} R(t)+\beta_{2} T(t)-d\right) \\
& >Q(t)\left(\beta_{2}\left(\frac{d}{\beta_{1}}-\varepsilon\right)+\beta_{2}\left(\frac{A}{d}-\frac{d}{\beta_{1}}-\varepsilon\right)-d\right) \\
& =Q(t)\left(\frac{A \beta_{2}}{d}-d-2 \beta_{2} \varepsilon\right),
\end{aligned}
$$

which is positive if $\varepsilon$ is small enough, as $r_{2}>1$, which contradicts $Q(t) \rightarrow 0$.

We have shown uniform weak persistence in all cases; to show uniform (strong) persistence, we apply Theorem 4.5 from [8]. Our flow is clearly continuous, the subspaces $X_{T}, X_{Q}, X \backslash X_{T}$ and $X \backslash X_{Q}$ are invariant. The existence of a compact attractor is also clear, as all solutions enter a compact region after some time. This means that all conditions of [8, Theorem 4.5] hold and thus we obtain uniform strong persistence.

\subsection{Global Stability}

\section{Theorem 3.2}

(1) Equilibrium $E_{R}$ is globally asymptotically stable if $r_{1}<1$ and $r_{2}<1$.

(2) Equilibrium $E_{T}$ is globally asymptotically stable on $X \backslash X_{T}$ if $r_{1}>1$ and $r_{2}<1 . E_{R}$ is globally asymptotically stable on $X_{T}$.

(3) Equilibrium $E_{Q}$ is globally asymptotically stable on $X \backslash X_{Q}$ if $r_{2}>1$ and $r_{3}<1 . E_{R}$ is globally asymptotically stable on $X_{Q}$ if $r_{1}<1$ and $E_{T}$ is globally asymptotically stable on $X_{Q}$ if $r_{1}>1$.

(4) Equilibrium $E_{T Q}$ is globally asymptotically stable on $X \backslash\left(X_{T} \cup X_{Q}\right)$ if $r_{2}>1$ and $r_{3}>1$. $E_{T}$ is globally asymptotically stable on $X_{Q}$ and $E_{Q}$ is globally asymptotically stable on $X_{T}$.

Proof First we note that the rodent subsystem (2) can be reduced to two dimensions by introducing the notation $F(t):=R(t)+T(t)$. We obtain the system

$$
\begin{aligned}
& F^{\prime}(t)=A-\beta_{2} F(t) Q(t)-d F(t), \\
& Q^{\prime}(t)=\beta_{2} F(t) Q(t)-d Q(t) .
\end{aligned}
$$

This system has two equilibria, $\left(\frac{A}{d}, 0\right)$ and $\left(\frac{d}{\beta_{2}}, \frac{A}{d}-\frac{d}{\beta_{2}}\right)$, with the latter one only existing if $r_{2}>1$. We use the Dulac function $1 / Q$ to show that there is no periodic solution of (4):

$$
\frac{\partial}{\partial F} \frac{A-\beta_{2} F Q-d F}{Q}+\frac{\partial}{\partial Q} \frac{\beta_{2} F Q-d Q}{Q}=-\beta_{2}-\frac{d}{Q}<0 .
$$


Thus, applying the Bendixson-Dulac criterion, we obtain that there is no periodic solution of (4), and by the Poincaré-Bendixson theorem we get that all solutions tend to an equilibrium.

In the first two cases, when $r_{2}<1$, only the first equilibrium exists. Thus, in this case $Q(t) \rightarrow 0$ and $F(t) \rightarrow \frac{A}{d}$ as $t \rightarrow \infty$, and therefore, the second equation of (2) takes the following form on the limit set:

$$
T^{\prime}(t)=\beta_{1}\left(\frac{A}{d}-T(t)\right) T(t)-d T(t)=\gamma T(t)-\beta_{1} T^{2}(t)
$$

with $\gamma=\left(\frac{A \beta_{1}}{d}-d\right)$.

The solution started from $T(t)=0$ is the constant solution $T(t) \equiv 0$, while the nontrivial solutions take the form

$$
\frac{C e^{\gamma t}}{1+\frac{\beta_{1}}{\gamma} C e^{\gamma t}}
$$

for $C \in \mathbb{R}_{+}$. Clearly, for $r_{1}<1$ (which is equivalent to $\gamma \leq 0$ ), the solutions tend to zero on the limit set, therefore, for all solutions, $T(t) \rightarrow 0$ as $t \rightarrow \infty$.

If $r_{1}>0$ (i.e. $\gamma>0$ ), we have $\lim _{t \rightarrow \infty} T(t)=\frac{A}{d}-\frac{d}{\beta_{1}}$ on the limit set; using the persistence of $T(t)$ we obtain that for all solutions, $T(t) \rightarrow \frac{A}{d}-\frac{d}{\beta_{1}}$ as $t \rightarrow \infty$.

In the case $r_{2}>1$, also the second equilibrium exists. However, we know from the previous subsection that for $r_{2}>1$, the compartment $Q(t)$ is uniformly persistent, so no solution with positive initial value in $Q(t)$ can tend to the first equilibrium. Thus, the limit of all such solutions is the second equilibrium and $Q(t) \rightarrow\left(\frac{A}{d}-\frac{d}{\beta_{2}}\right)$ as $t \rightarrow \infty$. We can proceed in a similar way as in the case $r_{2}<1$ : on the limit set, we can transform the second equation of (2) to

$$
\begin{aligned}
T^{\prime}(t) & =\beta_{1}\left(\frac{d}{\beta_{2}}-T(t)\right) T(t)-\beta_{2}\left(\frac{A}{d}-\frac{d}{\beta_{2}}\right) T(t)-d T(t) \\
& =\gamma T(t)-\beta_{1} T^{2}(t)
\end{aligned}
$$

with $\gamma=\left(\frac{d \beta_{1}}{\beta_{2}}-\frac{A \beta_{2}}{d}\right)$. Similarly as above, we can see that the solution started from $T(t)=0$ is the constant solution $T(t) \equiv 0$, while the nontrivial solutions take the form (5). In the case $r_{3}<1$ (which is equivalent to $\gamma \leq 0$ ), the solutions tend to 0 , while if $r_{3}>1$ (which is equivalent to $\gamma>0$ ), we have $\lim _{t \rightarrow \infty} T(t)=\frac{d}{\beta_{2}}-\frac{A \beta_{2}}{d \beta_{1}}$, and this is what we wanted to show.

Remark 3.1 We note that changing global asymptotic stability to attractivity, the results of Theorem 3.2 also hold when the given reproduction numbers are equal to 1 , instead of being smaller than 1 . 


\section{The Human Subsystem}

Let us now turn to the human subsystem of (1) consisting of the last three equations.

In the sequel, we assume that the rodent subsystem is in a steady state, and substitute any of the equilibria of the rodent subsystem into these equations to obtain the system

$$
\begin{aligned}
S^{\prime}(t)= & B-\eta_{1} T^{*} S(t)-\eta_{2} Q^{*} S(t)-v_{1} S(t) I(t)-v_{2} S(t) J(t) \\
& -\delta S(t)+\theta_{1} I(t)+\theta_{2} J(t) \\
I^{\prime}(t)= & \eta_{1} T^{*} S(t)+v_{1} S(t) I(t) \\
& -\eta_{2} Q^{*} I(t)-v_{2} J(t) I(t)-\delta I(t)-\theta_{1} I(t), \\
J^{\prime}(t)= & \eta_{2} Q^{*} S(t)+\eta_{2} Q^{*} I(t)+v_{2} S(t) J(t)+v_{2} I(t) J(t) \\
& -\delta J(t)-\rho J(t)-\theta_{2} J(t),
\end{aligned}
$$

where $T^{*}$ and $Q^{*}$ are the second, resp. third coordinates in any of the four equilibria (3).

To find all possible equilibria of (6), first we introduce the notation $G(t):=$ $S(t)+I(t)$ to obtain the system

$$
\begin{aligned}
G^{\prime}(t) & =B-\eta_{2} Q^{*} G(t)-v_{2} G(t) J(t)-\delta G(t)+\theta_{2} J(t), \\
J^{\prime}(t) & =\eta_{2} Q^{*} G(t)+v_{2} G(t) J(t)-\delta J(t)-\rho J(t)-\theta_{2} J(t) .
\end{aligned}
$$

We will apply the Bendixson-Dulac criterion with Dulac function $1 / J$ and the Poincaré-Bendixson theorem to obtain that in this case, all solutions of system (7) tend to one of the equilibria. Indeed, we have

$$
\begin{gathered}
\frac{\partial}{\partial G} \frac{B-\eta_{2} Q^{*} G-v_{2} G J-\delta G+\theta_{2} J}{J} \\
+\frac{\partial}{\partial J} \frac{\eta_{2} Q^{*} G+\nu_{2} G J-\delta J-\rho J-\theta_{2} J}{J} \\
\quad=-\frac{\eta_{2} Q^{*}}{J}-v_{2}-\frac{\delta}{J}-\frac{\eta_{2} G}{J^{2}}<0
\end{gathered}
$$

from which we obtain the assertion above.

This equation may have two equilibria:

$$
\left(\frac{D+B \nu_{2}-\sqrt{\left(D-B \nu_{2}\right)^{2}+4 B \eta_{2} Q^{*} \nu_{2}(\delta+\rho)}}{2 \delta \nu_{2}}, \frac{-D+B \nu_{2}+\sqrt{\left(D-B \nu_{2}\right)^{2}+4 B \eta_{2} Q^{*} \nu_{2}(\delta+\rho)}}{2(\delta+\rho) \nu_{2}}\right)
$$


and

$$
\left(\frac{D+B \nu_{2}+\sqrt{\left(D-B \nu_{2}\right)^{2}+4 B \eta_{2} Q^{*} \nu_{2}(\delta+\rho)}}{2 \delta \nu_{2}}, \frac{-D+B \nu_{2}-\sqrt{\left(D-B \nu_{2}\right)^{2}+4 B \eta_{2} Q^{*} \nu_{2}(\delta+\rho)}}{2(\delta+\rho) \nu_{2}}\right)
$$

denoted by $E_{1}$ and $E_{2}$, respectively, with $D=\delta^{2}+Q^{*} \eta_{2} \rho+\delta\left(Q^{*} \eta_{2}+\theta_{2}+\rho\right)$. The first coordinate of $E_{1}$ is always positive, since this coordinate may be rewritten as

$$
\frac{D+B v_{2}-\sqrt{\left(D+B \nu_{2}\right)^{2}-4 B \delta v_{2}\left(\delta+\theta_{2}+\rho\right)}}{2 \delta v_{2}} .
$$

It can easily be seen that the first coordinate of $E_{2}$ is always positive.

Let us first consider the case $Q^{*}>0$, (i.e. when the rodent subsystem tends to the equilibrium $E_{Q}$ or $E_{T Q}$, which is equivalent to $r_{2}>1$ ). In this case, the second coordinate of $E_{1}$ is always positive, while second coordinate of $E_{2}$ is negative if $Q^{*}>0$. Hence, in the case $Q^{*}>0$, there is only one equilibrium and using the Poincaré-Bendixson theorem, we obtain that all solutions tend to $E_{1}$.

In the case $r_{2} \leq 1$, i.e. when $Q^{*}=0$, the system takes the simpler form

$$
\begin{aligned}
G^{\prime}(t) & =B-v_{2} G(t) J(t)-\delta G(t)+\theta_{2} J(t), \\
J^{\prime}(t) & =v_{2} G(t) J(t)-\delta J(t)-\rho J(t)-\theta_{2} J(t) .
\end{aligned}
$$

This system has the two equilibria

$$
e_{1}:=\left(\frac{B}{\delta}, 0\right) \quad \text { and } \quad e_{2}:=\left(\frac{\delta+\theta_{2}+\rho}{\nu_{2}}, \frac{B \nu_{2}-\delta\left(\delta+\theta_{2}+\rho\right)}{\nu_{2}(\delta+\rho)}\right) .
$$

Now, it is easy to see that the first of these equilibria always exists, while the second one only exists if

$$
\mathcal{R}_{0}^{J}:=\frac{B v_{2}}{\delta\left(\delta+\theta_{2}+\rho\right)}>1
$$

Just as above, we obtain that all solutions of (8) tend to one of these equilibria. In the case $\mathcal{R}_{0}^{J} \leq 1$, this equilibrium is clearly $e_{1}$. Using similar methods as for the rodent subsystem, we will show that $J(t)$ is always uniformly strongly persistent if $\mathcal{R}_{0}^{J}>1$. To show this, we choose $\rho(x)=J$. Consider the extinction space $X_{J}$ defined as $X_{J}:=\left\{x \in \mathbb{R}_{+}^{2}: \rho(x)=0\right\}$; now $\Omega=M_{1}:=e_{1}$, which is obviously invariant, isolated and acyclic. Let us suppose that $M_{1}$ is not weakly $\rho$-repelling, i.e. there is a solution which tends to $e_{1}$ such that $J(t)>0$. Then, given any $\varepsilon>0$, for $t$ sufficiently large, we can give the following estimate for $J^{\prime}(t)$ :

$$
\begin{aligned}
J^{\prime}(t) & =v_{2} G(t) J(t)-\delta J(t)-\rho J(t)-\theta_{2} J(t) \\
& >J(t)\left(v_{2} \frac{B}{\delta}-v_{2} \varepsilon-\delta-\rho-\theta_{2}\right),
\end{aligned}
$$


which is positive as $\mathcal{R}_{0}^{J}>1$. Hence, in the case $\mathcal{R}_{0}^{J}>1$, all solutions of (8) started with positive initial value $J(0)$ tend to the equilibrium $e_{2}$.

We have now finished the analysis of (7) and showed that in each case, depending on the reproduction numbers $r_{2}$ and $\mathcal{R}_{0}^{J}$, all solutions of this equation tend to an equilibrium. Let us denote by $J^{*}$ the second coordinate of this equilibrium and substitute this value into the first two equations of (6) to obtain

$$
\begin{aligned}
S^{\prime}(t)= & B-\eta_{1} T^{*} S(t)-\eta_{2} Q^{*} S(t)-v_{1} S(t) I(t)-v_{2} J^{*} S(t) \\
& -\delta S(t)+\theta_{1} I(t)+\theta_{2} J^{*}, \\
I^{\prime}(t)= & \eta_{1} T^{*} S(t)+v_{1} S(t) I(t)-\eta_{2} Q^{*} I(t) \\
& -v_{2} J^{*} I(t)-\delta I(t)-\theta_{1} I(t) .
\end{aligned}
$$

This equation has a similar structure as (7). The two possible equilibria of system (9) are

$$
\left(\frac{v_{1}\left(B+\theta_{2} J^{*}\right)+P-\sqrt{\left(\nu_{1}\left(B+\theta_{2} J^{*}\right)-P\right)^{2}+H}}{2 v_{1} K}, \frac{\nu_{1}\left(B+\theta_{2} J^{*}\right)-P+\sqrt{\left(\nu_{1}\left(B+\theta_{2} J^{*}\right)-P\right)^{2}+H}}{2 v_{1} K}\right)
$$

and

$$
\left(\frac{\nu_{1}\left(B+\theta_{2} J^{*}\right)+P+\sqrt{\left(\nu_{1}\left(B+\theta_{2} J^{*}\right)-P\right)^{2}+H}}{2 v_{1} K}, \frac{\nu_{1}\left(B+\theta_{2} J^{*}\right)-P-\sqrt{\left(\nu_{1}\left(B+\theta_{2} J^{*}\right)-P\right)^{2}+H}}{2 v_{1} K}\right)
$$

denoted by $\mathcal{E}_{1}$ and $\mathcal{E}_{2}$, respectively, where the notations $K, P$ and $H$ are defined as $K=\left(\delta+\eta_{2} Q^{*}+v_{2} J^{*}\right), P=K\left(\delta+\theta_{1}+\eta_{1} T^{*}+\eta_{2} Q^{*}+v_{2} J^{*}\right)$ and $H=$ $4 \eta_{1} v_{1} T^{*}\left(B+\theta_{2} J^{*}\right) K$. Again, we can apply the Bendixson-Dulac criterion, in this case with the Dulac function $1 / I$, to show that all solutions tend to an equilibrium:

$$
\begin{gathered}
\frac{\partial}{\partial S} \frac{B-\eta_{1} T^{*} S-\eta_{2} Q^{*} S-v_{1} S I-v_{2} J^{*} S-\delta S+\theta_{1} I+\theta_{2} J^{*}}{I} \\
+\frac{\partial}{\partial I} \frac{\eta_{1} T^{*} S+v_{1} S I-\eta_{2} Q^{*} I-v_{2} J^{*} I-\delta I-\theta_{1} I}{I} \\
\quad=\frac{-\eta_{1} T^{*}}{I}-\frac{\eta_{2} Q^{*}}{I}-v_{1}-\frac{\nu_{2} J^{*}}{I}-\frac{\delta}{I}-\frac{\eta_{1} T^{*} S}{I^{2}}
\end{gathered}
$$

which is negative for all $I, S>0$.

Similarly as in the case of the equilibria of system (7), it is easy to see that the first coordinates of $\mathcal{E}_{1}$ and $\mathcal{E}_{2}$ are always positive, while the second coordinate of $\mathcal{E}_{2}$ is negative if $T^{*}>0$ (i.e. when $r_{1}>1$ and $r_{2}<1$, meaning that $E_{T}$ is globally asymptotically stable or $r_{2}>1$ and $r_{3}>1$ meaning that $E_{T Q}$ is globally asymptotically stable). Hence, in this case there is only one equilibrium, and by the Poincaré-Bendixson theorem, all solutions tend to this equilibrium. 
From the above, we obtain that if $T^{*}>0$ and $Q^{*}>0$, i.e. when $r_{2}>1$ and $r_{3}>1$, then all solutions tend to the equilibrium $\left(\mathcal{E}_{1}^{1}, \mathcal{E}_{1}^{2}, E_{1}^{2}\right)$, where upper index $i$ denotes the $i$ th coordinate of a given equilibrium.

In the case $T^{*}>0$ and $Q^{*}=0$, i.e. when $r_{1}>1$ and $r_{2} \leq 1, \mathcal{E}_{1}$ is the only equilibrium of (9), and the reproduction number $\mathcal{R}_{0}^{J}$ determines which equilibrium is the limit of the solutions of (8). Hence, if $r_{1}>1, r_{2} \leq 1$ and $\mathcal{R}_{0}^{J} \leq 1$ then all solutions of (6) tend to the equilibrium

$$
\left(\mathcal{E}_{1}^{1}, \mathcal{E}_{1}^{2}, 0\right)
$$

while if $r_{1}>1, r_{2} \leq 1$ and $\mathcal{R}_{0}^{J}>1$ then all solutions of (6) tend to the equilibrium

$$
\left(\mathcal{E}_{1}^{1}, \mathcal{E}_{1}^{2}, \frac{B \nu_{2}-\delta\left(\delta+\theta_{2}+\rho\right)}{\nu_{2}(\delta+\rho)}\right) .
$$

In the case $T^{*}=0$ (i.e. when $r_{1}<1$ and $r_{2}<1$, meaning that $E_{R}$ is globally asymptotically stable or $r_{2}>1$ and $r_{3}<1$, meaning that $E_{Q}$ is globally asymptotically stable), system (9) reduces to

$$
\begin{aligned}
S^{\prime}(t)= & B-\eta_{2} Q^{*} S(t)-v_{1} S(t) I(t)-v_{2} S(t) J^{*} \\
& -\delta S(t)+\theta_{1} I(t)+\theta_{2} J^{*}, \\
I^{\prime}(t)= & v_{1} S(t) I(t)-\eta_{2} Q^{*} I(t)-v_{2} I(t) J^{*}-\delta I(t)-\theta_{1} I(t),
\end{aligned}
$$

which has two equilibria

$\mathcal{E}_{1}=\left(\frac{\eta_{2} Q^{*}+\nu_{2} J^{*}+\delta+\theta_{1}}{\nu_{1}}, \frac{\nu_{1}\left(B+\theta_{2} J^{*}\right)-\left(\eta_{2} Q^{*}+\nu_{2} J^{*}+\delta\right)\left(\eta_{2} Q^{*}+\nu_{2} J^{*}+\delta+\theta_{1}\right)}{\nu_{1}\left(\eta_{2} Q^{*}+\nu_{2} J^{*}+\delta\right)}\right)$,

resp.

$$
\mathcal{E}_{2}=\left(\frac{B+\theta_{2} J^{*}}{\eta_{2} Q^{*}+\nu_{2} J^{*}+\delta}, 0\right) .
$$

One may easily observe that the second equilibrium always exists, while the sign of the second coordinate of the first equilibrium depends on the parameters and the limits $Q^{*}$ and $J^{*}$ : the first equilibrium exists if and only if

$$
\mathcal{R}_{0}^{I}:=\frac{\nu_{1}\left(B+\theta_{2} J^{*}\right)}{\left(\eta_{2} Q^{*}+v_{2} J^{*}+\delta\right)\left(\eta_{2} Q^{*}+\nu_{2} J^{*}+\delta+\theta_{1}\right)}>1 .
$$

In the case $\mathcal{R}_{0}^{I} \leq 1$, there is only one equilibrium, $\mathcal{E}_{2}$, so it is clear from the above that all solutions of (6) tend to the equilibrium 


$$
\left(\frac{B+\theta_{2} J^{*}}{\eta_{2} Q^{*}+v_{2} J^{*}+\delta}, 0, J^{*}\right) .
$$

In the case $\mathcal{R}_{0}^{I}>1$, we will again use persistence theory to show that all solutions of (10) tend to the equilibrium $\mathcal{E}_{1}$. We now choose $\rho(x)=I$ and consider the extinction space $X_{I}:=\left\{x \in \mathbb{R}_{+}^{2}: \rho(x)=0\right\}$. It is clear that now $\Omega=M_{1}:=\left\{\mathcal{E}_{2}\right\}$, which is invariant, acylic and isolated. Let us suppose that $M_{1}$ is not weakly $\rho$ repelling, i.e. there exists a solution which tends to $\mathcal{E}_{2}$ such that $I(t)>0$. Then, for any $\varepsilon>0$, for large enough $t$, we can estimate $I^{\prime}(t)$ as

$$
\begin{aligned}
I^{\prime}(t) & =I(t)\left(v_{1} S(t)-\eta_{2} Q^{*}-v_{2} J^{*}-\delta-\theta_{1}\right) \\
& >I(t)\left(v_{1}\left(\frac{B+\theta_{2} J^{*}}{\eta_{2} Q^{*}+v_{2} J^{*}+\delta}+\varepsilon\right)-\eta_{2} Q^{*}-v_{2} J^{*}-\delta-\theta_{1}\right),
\end{aligned}
$$

which is positive as $\mathcal{R}_{0}^{I}>1$. From this we obtain that in the case $\mathcal{R}_{0}^{I}>1$, all solutions of (10) started with positive initial value $I(0)$ tend to $\mathcal{E}_{1}$.

On the $\omega$-limit set of solutions of (6), Eq. (10) holds, which has at most two equilibria. Hence, the global attractor of (10) consists either of a single equilibrium or two equilibria and connecting orbits between them. When there is only one equilibrium, then the solutions of (6) tend to this equilibrium. When two equilibria exist, then $J(t)$ is uniformly persistent, hence, the $\omega$-limit set of positive solutions of (6) can only be the equilibrium with the positive $J$ coordinate.

Now we go through all possibilities regarding the value of $Q^{*}$ and $J^{*}$ to give a precise characterization. In the case $Q^{*}>0$ (i.e. $r_{2}>1$ ), there is only one equilibrium of (7), hence $J(t)$ tends to $E_{1}^{2}$. This means that in the case $r_{2}>1$ and $\mathcal{R}_{0}^{I} \leq 1$ all solutions of (6) tend to the equilibrium

$$
\left(\frac{B+\theta_{2} E_{1}^{2}}{\eta_{2}\left(\frac{A}{d}-\frac{d}{\beta_{2}}\right)+v_{2} E_{1}^{2}+\delta}, 0, E_{1}^{2}\right)
$$

while in the case $r_{2}>1$ and $\mathcal{R}_{0}^{I}>1$, all solutions of (6) tend to the equilibrium

$$
\left(\frac{\eta_{2} Q^{*}+v_{2} E_{1}^{2}+\delta+\theta_{1}}{v_{1}}, \frac{\nu_{1}\left(B+\theta_{2} E_{1}^{2}\right)-\left(\eta_{2} Q^{*}+\nu_{2} E_{1}^{2}+\delta\right)\left(\eta_{2} Q^{*}+\nu_{2} E_{1}^{2}+\delta+\theta_{1}\right)}{\nu_{1}\left(\eta_{2} Q^{*}+\nu_{2} E_{1}^{2}+\delta\right)}, E_{1}^{2}\right)
$$

with $Q^{*}=\left(\frac{A}{d}-\frac{d}{\beta_{2}}\right)$.

In the case $r_{2} \leq 1$ (i.e. $Q^{*}=0$ ), the reproduction number $\mathcal{R}_{0}^{J}$ determines the limit of $J(t)$. In the case $r_{1} \leq 1, r_{2} \leq 1, \mathcal{R}_{0}^{J} \leq 1, \mathcal{R}_{0}^{I} \leq 1$, all solutions of (6) tend to the equilibrium

$$
\left(\frac{B}{\delta}, 0,0\right)
$$


In the case $r_{1} \leq 1, r_{2} \leq 1, \mathcal{R}_{0}^{J} \leq 1, \mathcal{R}_{0}^{I}>1$, all solutions of (6) tend to the equilibrium

$$
\left(\frac{\delta+\theta_{1}}{v_{1}}, \frac{B}{\delta}-\frac{\delta+\theta_{1}}{v_{1}}, 0\right)
$$

In the case $r_{1} \leq 1, r_{2} \leq 1, \mathcal{R}_{0}^{J}>1, \mathcal{R}_{0}^{I} \leq 1$, all solutions of (6) tend to the equilibrium

$$
\left(\frac{\delta+\theta_{2}+\rho}{\nu_{2}}, 0, \frac{\nu_{2} B-\delta\left(\delta+\theta_{2}+\rho\right)}{\nu_{2}(\delta+\rho)}\right) .
$$

In the case $r_{1} \leq 1, r_{2} \leq 1, \mathcal{R}_{0}^{J}>1, \mathcal{R}_{0}^{I}>1$, all solutions of (6) tend to the equilibrium

$$
\left(\frac{\nu_{2} B+\theta_{1} \rho+\delta\left(\theta_{1}-\theta_{2}\right)}{\nu_{1}(\delta+\rho)}, \frac{\delta \theta_{2}-\nu_{2} B}{v_{1}(\delta+\rho)}+\frac{\delta+\theta_{2}+\rho}{\nu_{2}}-\frac{\theta_{1}}{\nu_{1}}, \frac{\nu_{2} B-\delta\left(\delta+\theta_{2}+\rho\right)}{\nu_{2}(\delta+\rho)}\right) \text {. }
$$

\section{Discussion}

We have established a six-compartment model to describe the spread of an infectious disease spread by ectoparasites which are transmitted to humans by rodents. We have identified three reproduction numbers for the rodent subsystem. These threshold numbers determine which of the four possible equilibria of the rodent subsystem is globally attractive. Assuming that the rodent subsystem is already in a steady state, we studied the human subsystem and calculated the possible equilibria of this subsystem depending on which of the rodent equilibria is globally attractive. We also determined which equilibrium of the human subsystem is globally attractive. Our results show that in each case, depending on the different reproduction numbers, one equilibrium is globally attractive. Our results show that if one type of the parasite (infectious or noninfectious) is present in the rodent population, then the same type will also be present in the human population. Using our results, we may study the possibilities of eradicating the disease. There are three main ways to control the disease: we may decrease the transmission rates $\eta_{1,2}$ between humans and rodents, increase the disinfestation rates $\theta_{1,2}$ of humans to shorten the duration of infestation of humans and we may reduce $d$ which means culling of the rodents.

Controlling only the human population (increasing the disinfestation rates $\theta_{1,2}$ ) only results in a mitigation not sufficient to eradicate the disease. The same holds for decreasing the transmission rates from rodents to humans, except the extreme case of decreasing the transmission rates $\eta_{1,2}$ to zero. In this latter case, one may decrease 
the human reproduction numbers to be less than 1 by increasing the disinfestation rates $\theta_{1,2}$ and thus eliminate the infestation.

By controlling the rodent population (increasing the death rate $d$ ), one can reduce the reproduction numbers $r_{1}$ and $r_{2}$ to be both less than 1 and this way one may eliminate the infestation among the rodents. Also in this case, infestation from rodents to humans can be eliminated and this way the human reproduction numbers determine which equilibrium of the human subsystem will be globally attractive. Hence, also in such a case, by increasing the disinfestation rate among humans may result in the elimination of the parasites and of the disease.

Acknowledgements A. Dénes was supported by Hungarian Scientific Research Fund OTKA PD and National Research, Development and Innovation Office NKFIH KH 125628 and the János Bolyai Research Scholarship of the Hungarian Academy of Sciences. G. Röst was supported by the EU-funded Hungarian grant EFOP-3.6.1-16-2016-00008 and Marie Sklodowska-Curie Grant No. 748193.

A. Dénes thanks to the International Union of Biological Sciences (IUBS) for partial support of living expenses in Moscow, during the 17th BIOMAT International Symposium, October 29November 04, 2017.

\section{References}

1. A. Dénes, G. Röst, Biomathematics 1, 1209256, 1-5 (2012)

2. A. Dénes, G. Röst, Nonlinear Anal. Real World Appl. 18, 100-107 (2014)

3. A. Dénes, G. Röst, Impact of excess mortality on the dynamics of diseases spread by ectoparasites, in Interdisciplinary Topics in Applied Mathematics, Modeling and Computational Science, ed. by M. Cojocaru, I.S. Kotsireas, R.N. Makarov, R. Melnik, H. Shodiev. Springer Proceedings in Mathematics \& Statistics, vol. 117 (Springer, Cham, 2015), pp. 177-182

4. M.R. Holbrook, J.F. Aronson, G.A. Campbell, S. Jones, H. Feldmann, A.D.T. Barrett, J. Infect. Dis. 191, 100-108 (2005)

5. M.J. Keeling, C.A. Gilligan, Proc. R. Soc. B 267, 2219-2230 (2000)

6. Ch.D. Paddock, M.E. Eremeeva, Rickettsialpox, in Rickettsial Diseases, ed. by D. Raoult, Ph. Parola (CRC Press, New York, 2007)

7. H.L. Smith, An Introduction to Delay Differential Equations with Applications to the Life Sciences (Springer, New York, 2011)

8. H.L. Smith, H.R. Thieme, Dynamical Systems and Population Persistence (AMS, Providence, 2011)

9. Y. Tselentis, A. Gikas, Murine typhus, in Rickettsial Diseases, ed. by D. Raoult, Ph. Parola (CRC Press, New York, 2007)

10. G. Watt, P. Kantipong, Orientia tsutsugamushi and scrub typhus, in Rickettsial Diseases, ed. by D. Raoult, Ph. Parola (CRC Press, New York, 2007) 